\title{
Perché è utile tradurre gli anglismi
}

\section{Claudio Marazzini}

PUBBLICATO: 16 MAGGIO 2021

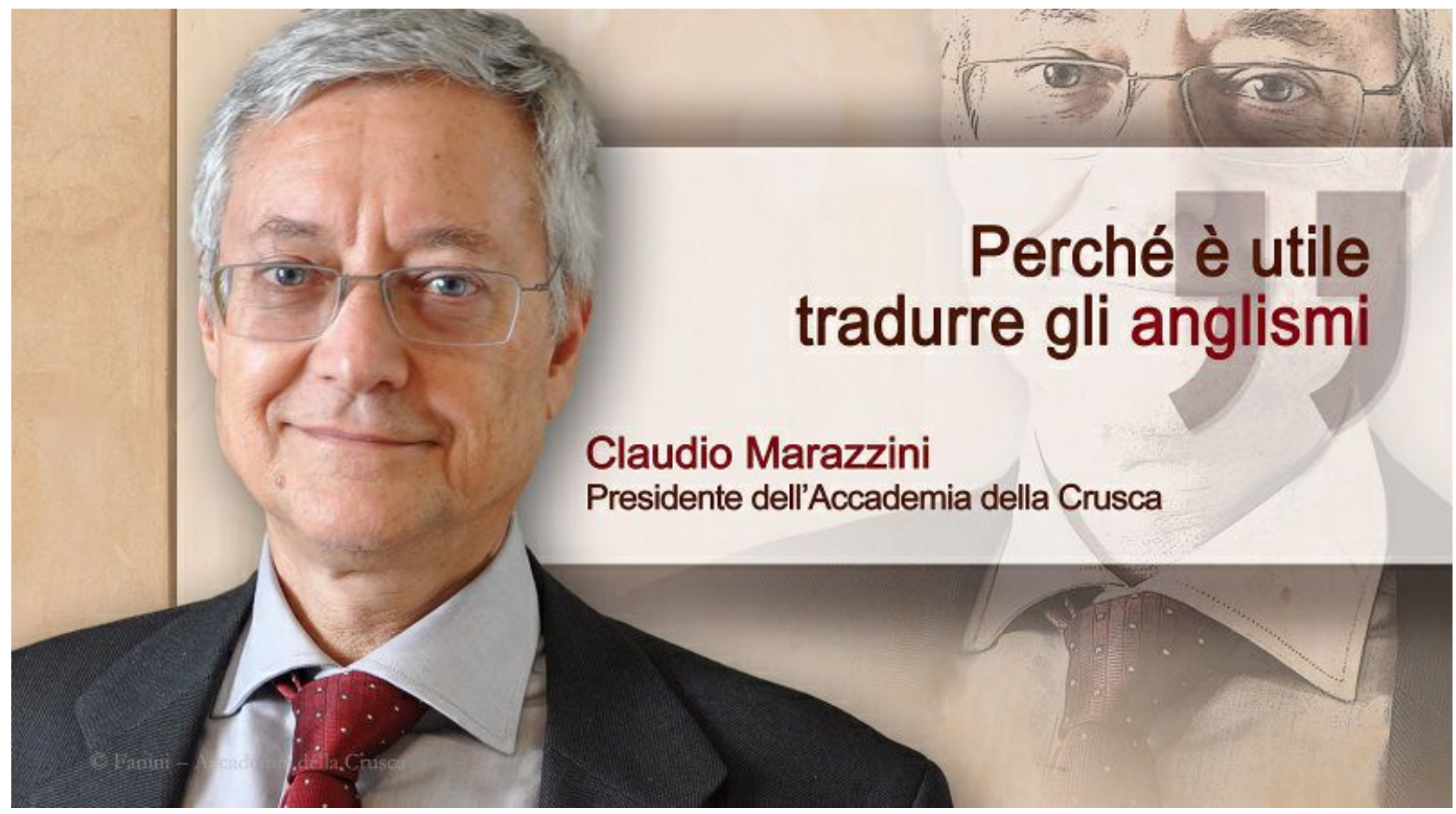

$\mathrm{M}$

i sono imbattuto, nella rivista "Trend online", in un articolo dal titolo Conto corrente out per tutti! Il dramma! Come molti interventi di tema economico, offre una buona campionatura di anglismi, sul significato dei quali è lecito interrogarsi, non necessariamente in una prospettiva passatista, puristica, o di difesa della bellezza della lingua. Già titolo della rivista contiene Trend, "andamento", "tendenza", ma l'anglismo è pressoché obbligato nel contesto tecnico-economico del linguaggio della finanza. Il titolo dell'articolo, che pur utilizza espedienti di letteratura da puntini (l'esclamativo doppio e la parola "dramma", giocata come spinta verso una reazione emozionale), esibisce l'anglismo out per "essere fuori", nel significato informale in inglese di 'at an end', o addirittura 'no longer alight; extinguished', come spiega l'Oxford dictionary. L'anglismo funziona bene per la sua brevità, nel contesto già tematicamente predisposto al forestierismo.

L'articolista tratta la possibile eliminazione dei bancomat, e poi cita in un riquadro alcune dichiarazioni di un dirigente finanziario, riportate a mo' di virgolettato, ma senza virgolette. Nella citazione si noterà un refuso (non l'ho corretto, ma l'ho segnalato con un sic). Si manifesta cosi una tendenza tipica del linguaggio della Rete, in cui la fretta non lascia tempo alla cura formale, e anzi il copia-incolla moltiplica e diffonde le imperfezioni: prova ne sia che il motore di ricerca di Google mi ha permesso di trovare immediatamente altri due siti che riportavano la medesima sconcordanza nella medesima dichiarazione (in questo caso tra virgolette), a riprova di un fatto del resto ben noto: in Rete accade che si scriva e si copi molto, ma si (ri)legge poco o nulla di ciò che si scrive. Se non fosse cosi, un errore del genere sarebbe stato facilmente individuato e rapidamente corretto, tanto piu che il correttore automatico di Word lo individua subito e lo evidenzia con due sottolineature azzurre, come posso verificare sul mio schermo mentre scrivo. Leggiamo questo virgolettato (non virgolettato 
dal giornale on line, forse per pigra omissione). Vi si riporta la dichiarazione di un alto dirigente bancario, decisiva per il tema trattato nell'articolo di Trend on line (sostituisco i nomi con asterischi):

Stiamo evolvendo verso un modello cashless e sempre più mobile-first. Questo per rispondere alla preferenze [sic!] dei nostri clienti, il $96 \%$ dei quali opera solo tramite canali digitali, $7 \mathrm{su}$ Io prediligendo lo smartphone, ha spiegato $* * *$, country manager di $* * *$.

Mi importa osservare che gli anglismi assumono una funzione che supera la distinzione tra prestiti di necessità o di lusso, e anche quella di "doni" (gradita ai linguisti più liberali), e assume una funzione eminente di carattere retorico-formale. Concediamoci una breve analisi che vuole essere "demistificatoria", come si sarebbe detto alcuni anni fa.

Parla il country manager della banca: l'anglismo può apparire un prestito di lusso; è interpretabile come "direttore generale nazionale per l'Italia"; tuttavia la qualifica inglese è quella ufficiale, che compare anche nel sito della banca, dov'è collocato il profilo del dirigente. Dunque potremmo dire che si tratta di linguaggio aziendale di una banca internazionale; "direttore nazionale" potrebbe far pensare che esista un direttore internazionale, di rango superiore, e che le decisioni siano state prese a livello globale, non necessariamente per interesse dell'Italia. In questo senso, "country" è più comodo di "nazionale". Un effetto curioso e fuorviante, in questo contesto bancario, è dovuto al fatto che il parlante italiano è tradizionalmente abituato connotare il termine country nel senso della musica country, in quanto legato alla musica popolare americana. Quanto al costrutto, la produttività di $x+$ manager è assodata: i treni di Italo, ad esempio, hanno rimpiazzato il capotreno con il train manager, da collocare nella serie general manager, railway manager, training manager, disaster manager, emergency manager ecc.

Il Country manager di *** emette dunque un comunicato in cui gli anglismi si combinano con le parole italiane per conseguire un fine retorico ed emotivo: la banca sta evolvendo verso qualche cosa di nuovo. Il verbo non è neutrale: l'evoluzione è sempre di segno positivo, mentre un generico cambiamento potrebbe essere negativo o sgradevole. Il verbo in incipit segna senza esitazione la positività dell'evento incipiente: la scelta, in quanto evolutiva, è di per sé inevitabile, ma inoltre è necessaria, perché determinata dalla risposta a esigenze dei clienti, non da essi dichiarate, ma oggettivamente dedotte da numeri, cioè fondate su dati statistici, assunti come certi e indiscutibili. Ecco l'oggettività della matematica, oggi largamente e generosamente adoperata nei più diversi contesti. Grazie ai dati, una decisione aziendale è dimostrata quale adesione alla volontà dei clienti. Qui si inserisce assai bene la funzione speciale dei due anglismi, entrambi abbinati al medesimo sostantivo italiano, modello, parola già di per sé positiva. Un modello è di per sé positivo perché si propone all'imitazione. Un modello è positivo anche perché è strutturale, cioè implica una programmazione. Un modello rassicura, perché non è un semplice tentativo, scommessa incerta. Tale modello risulta qualificato dagli aggettivi inglesi cashless e mobile first. Cashless ha avuto grande fortuna anche per le politiche antievasione progettate dal governo italiano, che demonizzano il cash, mentre il less e sempre eliminazione di qualche cosa di negativo, o comunque segno di un vantaggio tecnologico: wireless è il capofila di questa rivoluzione. Quanto a mobile, gode di immensa fortuna grazie al successo complessivo della telefonia mobile. L'uso di /'məubail/ rispetto al graficamente identico italiano mobile non ha alcuna funzione razionale, scientifica o comunicativa, ma solo suggestiva, tanto che la parola potrebbe essere italiana, ma l'ordine ne certifica la natura inglese, assunta come promozione, nobilitazione nella lingua internazionale tecnologicamente "superiore". Quanto al costrutto x + first, esso gode di immensa fortuna, in concorrenza all'italiano prima + x (cfr. ad es. il ben noto "prima gli italiani"); ma ultimamente il "progressismo" della formula ha acquisito punti a proprio favore con l'"America First" per il cosiddetto sovranismo vaccinale attribuito ora persino, dopo l'era di Trump, a 
un presidente democratico come Biden (ad es. https://www.project-syndicate.org/commentary/toomuch-continuity-between-trump-biden-foreign-policies-by-melvyn-krauss-202I-05

https://www.lavocedinewyork.com/news/politica/202I/03/30/piano-di-rilancio-economico-con-bidensi-passa-da-american-first-ad-american-made/). Il messaggio della banca si presenta dunque come ben modulato per apparire progressista, necessario, gradito, incisivo, e gli anglismi si inseriscono perfettamente nella corrispondente costruzione retorica, in cui ha una funzione di rilievo anche la scelta di opportune parole italiane, come abbiamo visto.

Una parafrasi con traduzione dei forestierismi, però, comunica meglio il contenuto del messaggio:

Abbiamo deciso di imporre un modello senza soldi contanti e che costringa prima di tutto all'uso del telefono cellulare nel rapporto con la banca e con il denaro, anche perché lo si può fare, in quanto risulta dalle nostre ricerche di mercato che i nostri clienti operano già al $96 \%$ solo tramite canali digitali, 7 su Io prediligendo il telefono cellulare - ha spiegato $* * * *$, Direttore nazionale per l'Italia di $* * *$.

È per questo che io consiglio sempre di tradurre, perché tradurre vuol dire chiarire e riflettere, mettendo argine alla banalità dei luoghi comuni.

\section{Cita come:}

Claudio Marazzini, Perché è utile tradurre gli anglismi, "Italiano digitale", XVII, 2021/2 (aprilegiugno)

DOI: $10.35948 / 2532-9006 / 2021.8544$

Copyright 2021 Accademia della Crusca

Pubblicato con licenza creative commons CC BY-NC-ND 